University of Wollongong

Research Online

Faculty of Engineering and Information

Faculty of Engineering and Information

Sciences - Papers: Part A

Sciences

$1-1-2014$

\title{
Shape optimization of thin-walled steel sections using graph theory and ACO algorithm
}

\author{
Pezhman Sharafi \\ University of Wollongong, psharafi@uow.edu.au \\ Lip H. Teh \\ University of Wollongong, Iteh@uow.edu.au \\ Muhammad N. S Hadi \\ University of Wollongong, mhadi@uow.edu.au
}

Follow this and additional works at: https://ro.uow.edu.au/eispapers

Part of the Engineering Commons, and the Science and Technology Studies Commons

Research Online is the open access institutional repository for the University of Wollongong. For further information contact the UOW Library: research-pubs@uow.edu.au 


\title{
Shape optimization of thin-walled steel sections using graph theory and ACO algorithm
}

\begin{abstract}
This paper presents an intuitive procedure for the shape and sizing optimizations of open and closed thinwalled steel sections using the graph theory. The goal is to find shapes of optimum mass and strength (bi-objectives). The shape optimization of open sections is treated as a multi-objective all-pairs shortest path problem, while that of closed sections is treated as a multi-objective minimum mean cycle problem. The sizing optimization of a predetermined shape is treated as a multi-objective single-pair shortest path problem. Multi-colony ant algorithms are formulated for solving the optimization problems. The verification and numerical examples involving the shape optimizations of open and closed thin-walled steel sections and the sizing optimization of trapezoidal roof sheeting are presented.
\end{abstract}

\section{Keywords}

thin, optimization, shape, aco, theory, walled, algorithm, graph, sections, steel

\section{Disciplines}

Engineering | Science and Technology Studies

\section{Publication Details}

Sharafi, P., Teh, L. H. \& Hadi, M. N. S. (2014). Shape optimization of thin-walled steel sections using graph theory and ACO algorithm. Journal of Constructional Steel Research, 101 331-341. 


\title{
Shape Optimisation of Thin-Walled Steel Sections using Graph Theory and ACO Algorithm
}

\author{
P. Sharafi ${ }^{\mathrm{a}}$, Lip H. Teh ${ }^{\mathrm{b} *}$, Muhammad N. S. Hadi ${ }^{\mathrm{c}}$ \\ ${ }^{a}$ Research Associate, School of Civil, Mining \& Environmental Engineering, University of Wollongong, \\ Wollongong, NSW 2522, Australia. \\ ${ }^{\mathrm{b}}$ Senior Lecturer, School of Civil, Mining \& Environmental Engineering, University of Wollongong, \\ Wollongong, NSW 2522, Australia. \\ ${ }^{\mathrm{c}}$ Associate Professor, School of Civil, Mining \& Environmental Engineering, University of Wollongong, \\ Wollongong, NSW 2522, Australia. \\ ${ }^{*}$ Corresponding author at School of Civil, Mining \& Environmental Engineering, University of Wollongong, \\ Wollongong, NSW 2522, Australia. Tel: +61242213564, fax: +61242213238, e-mail: 1teh@uow.edu.au.
}

\begin{abstract}
This paper presents an intuitive procedure for the shape and sizing optimisations of open and closed thin-walled steel sections using the graph theory. The goal is to find shapes of optimum mass and strength (bi-objectives). The shape optimisation of open sections is treated as a multi-objective all-pairs shortest path problem, while that of closed sections is treated as a multi-objective minimum mean cycle problem. The sizing optimisation of a predetermined shape is treated as a multi-objective single-pair shortest path problem. Multi-colony ant algorithms are formulated for solving the optimisation problems. The verification and numerical examples involving the shape optimisations of open and closed thin-walled steel sections and the sizing optimisation of trapezoidal roof sheeting are presented.
\end{abstract}

Keywords: thin-walled section, shape optimisation, sizing optimisation, graph theory. 


\section{Introduction}

Thin-walled steel sections, whether hot-rolled or cold-formed, are well-established construction products that have been widely used in various structural systems owing to their versatility in design, economical production and fast installation. Finding optimum shapes of thin-walled steel sections is currently a problem of strong interest, where optimisation is aimed at achieving efficient use of the steel material either by maximising the desirable properties of the section for a given mass or by minimising the mass for a given application.

With regard to cold-forming, the sheet steel can be formed into many shapes to suit structural and constructional requirements. This is particularly true for the newly invented method called chain-die forming [1]. Chain-die forming is not only able to form shapes that are not feasible with the traditional roll forming method, but also results in negligible residual stresses. It therefore opens up the possibility of forming new shapes that have not been previously considered. The primary objective should therefore be discovering optimum shapes to suit particular applications rather than determining optimum dimensions of standard shapes, which can now be achieved with the aid of the Direct Strength Method [2]. If the design search space is limited to one or a number of predetermined shapes, it is highly probable that the search result will be sub-optimal.

While the design variables in sizing optimisation are the dimensions of a predetermined shape [3-12], the vector of design variables in shape optimisation represents the boundary of the structural domain [13-17]. A major challenge in shape optimisation is the large number of design variables and constraints that need to be taken into account. Choosing an appropriate shape from a large number of possible shapes and dimensions entails a large combinatorial optimisation problem that is discrete in nature.

The structural performance of a thin-walled steel section depends not only on the characteristics of their components, but also on their relative locations and connectivity (topology). In this regard, graph theory based methods are powerful means to represent structural systems so that their geometry and topology can be understood clearly [18-23]. Graph theory based methods are readily formulated for a wide range of structural problems as a result of interaction with other fields of mathematics, and can be applied to a wide range of combinatorial optimisation problems [21-26]. 
This paper presents the graph theory approach for optimum shape discovery of open and closed thin-walled steel sections. The section strength is used as a generic term, and depending on the instance may mean the strength with respect to compression, flexural, torsional or shear action effects. While the present study does not consider the issues of various buckling modes as they are outside the scope of the paper, the concept is capable of incorporating them.

There are a number of methods available for dealing with discrete optimisation problems, such as the branch and bound method, simulated annealing, genetic algorithm and ant colony optimization. However, the Ant Colony Optimisation (ACO) algorithm is particularly suited to the present study since it has been proven to be one of the most robust stochastic metaheuristics for solving large combinatorial optimisation problems which can be reduced to finding the shortest paths through graph theory models [27]. This biology-inspired algorithm is very suitable for modeling geometry related optimization problems [26, 28-29].

This paper formulates ACO algorithms for shape optimisations of open and closed thinwalled steel sections based on the graph theory approach. Verification and numerical examples are included to demonstrate the application of the presented methodology.

\section{The Graph Theory}

One advantage of the graph theory approach to optimisation problems is that a continuous optimisation problem can be transformed into a discrete one, where the variables belonging to the space $R^{\mathrm{n}}$ are finite dimensional. In fact, the graph theory approach can be considered as a link between discrete spaces and continuous ones. By employing the graph theory, the shape optimisation problem becomes a combinatorial optimisation problem of discrete space.

In general, an instance of a combinatorial optimisation problem $\Pi$ is a triple $(S, f, \Omega)$, in which $S$ is the set of candidate solutions, $f$ is the objective function that assigns an objective function value $f(s)$ to each candidate solution $s \in S$, and $\Omega$ is a set of constraints. A feasible

solution is one that belongs to the set $\hat{S} \subseteq S$ of candidate solutions and satisfies the constraints $\Omega$. The goal is to find the globally optimum solution among feasible solutions $S^{*} \in \hat{S}$. 


\subsection{Definition of terms}

A graph $G(N, E)$ consists of a set of nodes $N$ and a set of edges $E$, with a relation of incidence that associates each edge with a pair of nodes as its ends. As shown in Fig. 1, a path $P$ of graph $G$ is a finite sequence whose terms are alternately nodes and edges, in which no edge or node appears more than once. A cycle $C$ is a path for which the starting node and the ending node are the same; i.e. a cycle is a closed path. The length of a path (or cycle) $L$ is the number of its edges.

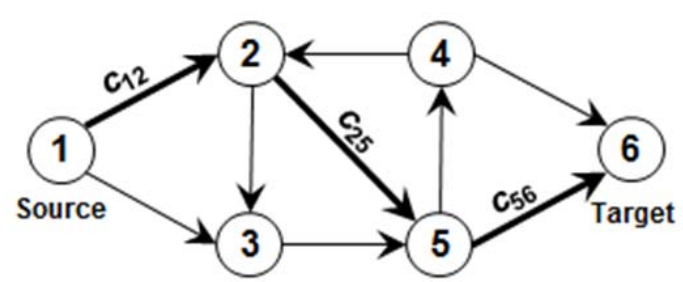

a) A path and the corresponding cost: $P=\left[n_{1}, e_{12}, n_{2}, e_{25}, n_{5}, e_{56}, n_{6}\right]$ cost: $L(P)=C_{12}+C_{25}+C_{56}$

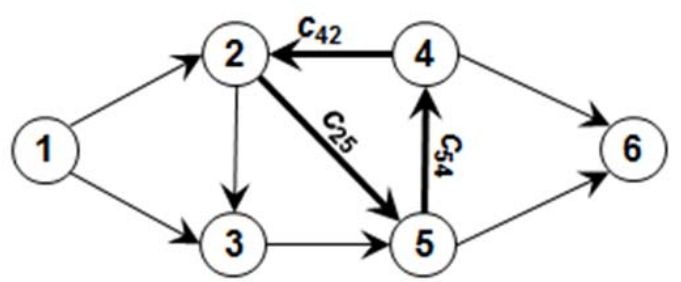

b) A cycle and the corresponding cost: $P=\left[n_{2}, e_{25}, n_{5}, e_{54}, n_{4}, e_{42}, n_{2}\right]$ cost: $L(\Gamma)=c_{25}+c_{54}+c_{42}$

Fig. 1 A path and a cycle on directed graphs

\subsection{Multi-Objective Shortest Path Problem}

In the graph theory, the shortest path problem is the problem of finding a path from a specified node called the source, to a second specified node, called the destination (or target), such that the sum of the weights (or lengths) of its constituent edges is minimised. It is relevant to a wide variety of real world applications, such as in telephone routing, material distribution, salesperson routing, investment strategies and personnel scheduling. The shortest path problem is an NP-hard combinatorial optimisation problem, which means that it is strongly believed that they cannot be solved to optimality within polynomially bounded computation time. To practically solve large instances, one often has to use heuristics that returns near-optimal solutions in a relatively short time [30].

Consider a weighted undirected graph $G(N, E)$. Let the number of nodes $n=|N|$ and the number of edges $m=|E|$. Each edge $e_{i j} \in E$ is assigned a cost (or length) of $c_{i j}$. If $c_{i j}$ has multiple criteria, the problem is called a multi-objective shortest path problem. In this case the edge $e_{i j}$ has associated values ${ }^{k} c_{i j}$, in which $k \in\{1,2, \ldots, r\}$, for each criterion $k$. 
Obviously, for undirected graphs ${ }^{k} c_{i j}={ }^{k} c_{j i}$. The adjacency set $A(i)$ for node $i$ is the set of all edges incident from $i$, that is $A(i)=\{(i, j) \mid(i, j) \in E\}$. The integer programming formulation for the multi-objective shortest path problem from Node $s$ to Node $t$ can be given as follows [31]:

$$
\begin{gathered}
\min f=\left(\sum_{i, j \in V}{ }^{1} c_{i j} e_{i j}, \sum_{i, j \in V}{ }^{2} c_{i j} e_{i j}, \ldots, \sum_{i, j \in V}{ }^{r} c_{i j} e_{i j}\right) \\
\forall e_{i j} \in E: e_{i j}= \begin{cases}1 & \text { if Edge } e_{i j} \text { is chosen } \\
0 & \text { if Edge } e_{i j} \text { is notchosen }\end{cases} \\
\forall i \in N-\{s, t\}: \sum_{j} e_{i j}-\sum_{k} e_{k i}=0 \\
\forall i, j \in N: \sum_{i} e_{s i}-\sum_{j} e_{j s}=1 \\
\forall i, j \in N: \sum_{i} e_{i t}-\sum_{j} e_{t j}=1
\end{gathered}
$$

Equation (1) represents the objective and Eq. (2) defines the binary variable $e_{i j}$. Equations (3) through (5) are the constraints.

Equation (3), as an ordinary flow conservation constraint, states that for all nodes except for the source and target points, the edges leaving them are equal to the edges entering them. Equations (4) and (5) state that the difference between the number of edges leaving the source and target points and the number of edges entering them, respectively, is one. In other words, the edges are not on a cycle. In a generalised form of the problem, called the all-pairs shortest path problem, the shortest paths from every possible source to every possible target are determined in order to form the shortest path matrix that gives the shortest path between every pair of vertices.

\subsection{Multi-Objective Minimum Mean Cycle Problem}

The minimum mean cycle problem is an NP-hard classical problem in combinatorial optimisation and has many applications in goods distribution and transportation networks [32]. In the minimum mean cycle problem, the goal is to find a cycle whose ratio of length (or cost) to number of arcs is the minimum. 
For an undirected graph $G=(N, E)$, each edge $e_{i j} \in E$ is assigned multi criteria $\operatorname{cost}^{k} c_{i j}$, in which $k \in\{1,2, \ldots, r\}$. The aim is to find a cycle $\Gamma$ that minimises the mean cost of the cycle. The multi-objective minimum mean cycle problem can be formulated as:

$$
\begin{gathered}
\min f=\frac{1}{|\Gamma|}\left(\sum_{i, j \in V}{ }^{1} c_{i j} e_{i j}, \sum_{i, j \in V}{ }^{2} c_{i j} e_{i j}, \ldots, \sum_{i, j \in V}{ }^{r} c_{i j} e_{i j}\right) \\
\forall e_{i j} \in E: e_{i j}= \begin{cases}1 & \text { if Edge } e_{i j} \text { is chosen } \\
0 & \text { if Edge } e_{i j} \text { is notchosen }\end{cases} \\
\forall i \in N: \sum_{j} e_{i j}-\sum_{k} e_{k i}=0
\end{gathered}
$$

Equation (6) is the objective and Eq. (8) is an ordinary flow conservation constraint stating that for all nodes, edges leaving them are equal to the edges entering them. It guarantees that the selected edges are on a cycle.

\section{$3 \quad$ Problem Definitions}

Consider a cross-section of an arbitrary shape (whether open or closed section) lying in the $x$ $y$ plane under a general set of actions resulting in compression, flexural, torsional and shear action effects, as shown in Fig. 2. The cross-section, defined by $n$ nodes and $m$ elements (edges) connecting the nodes, has a uniform and constant wall thickness. The problem is to find the shape that has the optimum mass and section strength. As previously mentioned, "section strength" is a generic term that corresponds to the imposed action effects.

In the present problem, to minimise the mass is to minimise the cross-section area $A$. Since the thickness of the cross-section is uniform and constant, the mass minimisation of the section reduces to the length minimisation of the section. The dimensional constraints on the elements (edges) of a cross-section may be defined considering the effective width (or depth) for the elements [33]. Construction and manufacturing constraints may also be applied to the cross-sectional dimensions. 


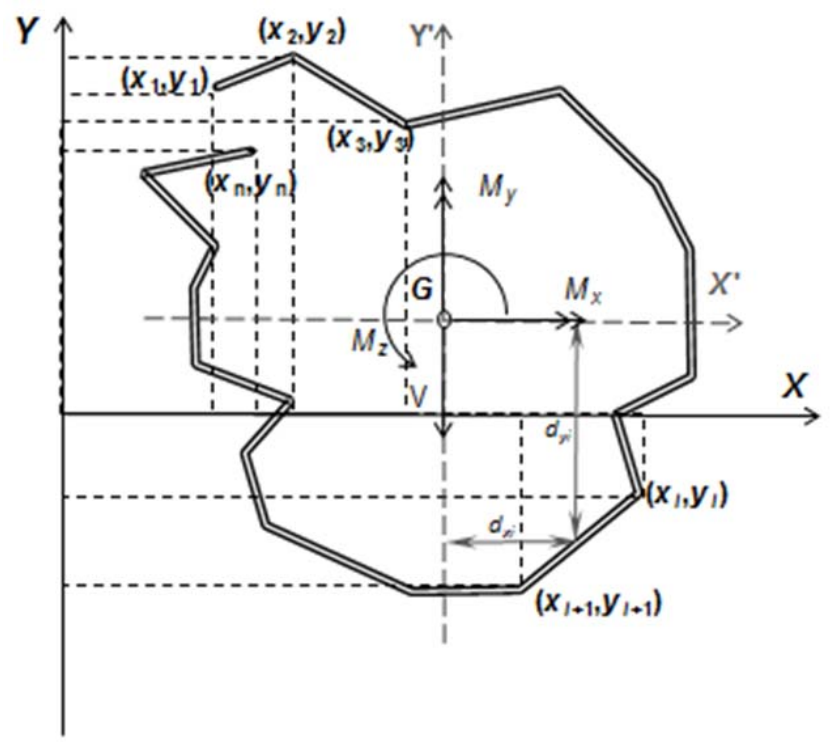

Fig. 2 Nodal coordinates of an arbitrary cross-section of constant thickness

Some design standards including AS/NZ 4600 [33] specify that the section properties such as the second moment of area and the torsion constant are to be determined by discretising the cross-section into small elements. In the present case, the section properties can be determined from the nodal coordinates used to define the cross-section. Since the section properties are functions of the nodal coordinates and the connectivity between the nodes, the shape optimisation problem becomes the problem of determining the optimum nodal coordinates and connectivity on the $x-y$ plane that result in the optimum total length of the elements and section strength.

The first steps in an optimisation problem are to identify the design variables and to define the related state variables. In the present problem, the variables are the nodal coordinates of the cross-section and their connectivity that turn into discrete and binary variables, respectively, by being mapped onto a graph. The idea is to represent a cross-section as a mathematical graph, which is made up of members (sub-graphs) having one-to-one relationship with the physical design. Any changes to the graph reflect same to the crosssection, so the shape optimisation of the graph is equivalent to the shape optimisation of the cross-section.

For an arbitrary cross-sectional shape of constant thickness $t$ depicted in Fig. 2, which comprises nodes $N_{1}$ through $N_{n}$ located on the coordinates $\left(i_{1}, j_{1}\right)$ through $\left(i_{n}, j_{n}\right)$, the cross- 
sectional area $A$, the centroid coordinates $x_{\mathrm{G}}$ and $y_{\mathrm{G}}$. and the second moments of area $I$ can be determined using Eqs. (9) through (11), respectively [14]:

$$
\begin{aligned}
& A=t \sum_{i=1}^{n-1} \sqrt{\left(x_{i+1}-x_{i}\right)^{2}+\left(y_{i+1}-y_{i}\right)^{2}} \\
& \left\{\begin{array}{l}
x_{G}=\frac{t}{A} \sum_{i=1}^{n-1} \frac{\left(x_{i+1}+x_{i}\right) l_{i}}{2} \\
y_{G}=\frac{t}{A} \sum_{i=1}^{n-1} \frac{\left(y_{i+1}+y_{i}\right) l_{i}}{2}
\end{array}\right. \\
& \left\{\begin{array}{c}
I_{G X}=\sum_{i=1}^{m}\left(I_{X_{i}}+l_{i} d_{X_{i}}^{2}\right) \\
I_{G Y}=\sum_{i=1}^{m}\left(I_{Y_{i}}+l_{i} d_{Y_{i}}^{2}\right)
\end{array}\right.
\end{aligned}
$$

where $d_{X_{i}}\left(\right.$ or $d_{Y_{i}}$ ) are the distance between the principal axis $X($ or $Y)$ and the centroid of the $i$ th edge. The variables $I_{X_{i}}$ and $I_{Y_{i}}$ are the second moments of area of the $i$ th edge about its local axes. Other geometric properties of the cross-section can be determined in a similar manner.

The present approach comprises defining the graph representing the section being optimised, and applying the corresponding graph theory. Consider a graph $G(N, E)$ having a diagonal grid pattern in which every pair of distinct nodes are connected by a unique edge, as shown in Fig. 3. The graph is formed in such a way that every node or edge on the graph is a potential node or edge of a cross-section. The length and width of the graph are defined to be equal to the maximum permitted dimensions of the optimised section. The minimum distance between two adjacent nodes is equal to the required accuracy $\varepsilon$. Since an increase in accuracy (smaller $\varepsilon)$ entails more computation costs, a balance must be stroke between accuracy and computation costs. 


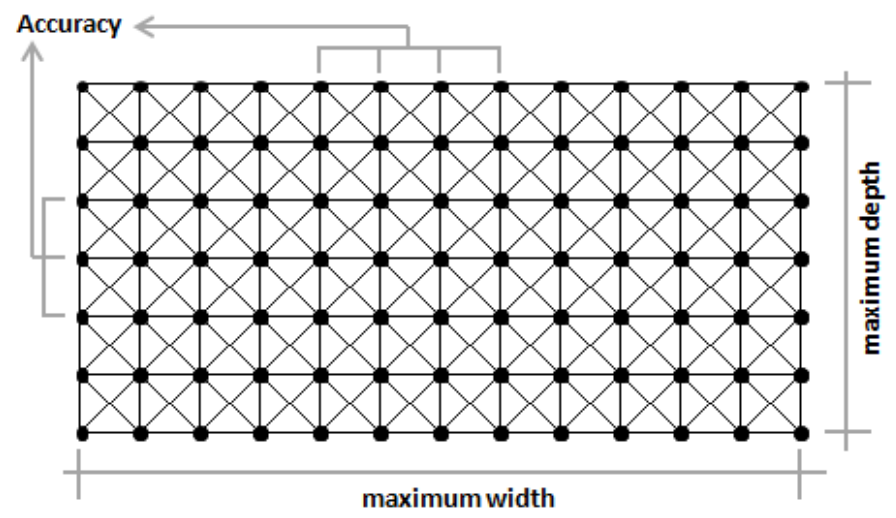

Fig. 3 A grid pattern for shape optimisation

\subsection{Shape optimisation of an open section}

The shape optimisation of an open section is treated as an all-pairs shortest path problem, which is represented by Eq. (1). Each edge on the graph is assigned a cost equal to its length $L(P)$, and a path is assigned a cost equal to the inverse of the section strength $1 / S_{G}$. A path therefore encompasses two cost criteria. For example, in the optimisation of a section for flexural rigidity, each path is assigned two costs equal to its total length and to the inverse of its second moment of area, respectively. The objective function for shape optimisation of an open section is formulated as follows:

$$
f_{P}=\left(L\left(P_{i}\right), \frac{1}{S_{G_{i}}}\right) \quad i \in \text { All-pairs path }
$$

The shape optimisation of an open section in the present study becomes a bi-objective allpairs shortest path problem, in which the objectives are functions of the nodal coordinates. Both objectives are scalar functions that depend on discrete design variables.

\subsection{Shape optimisation of a closed section}

The shape optimisation of a closed section is treated as a minimum mean cycle problem, which is represented by Eq. (6). In order to be consistent with the standard formulation of the minimum mean cycle problem, each edge on the graph is assigned a cost equal to the square of its length $L^{2}(I)$, and a cycle is assigned a cost equal to the product of its length and the inverse of the section strength $L(\Gamma) / S_{G}$. The resulting objective function for shape optimisation of a closed section is thus: 


$$
f_{\Gamma}=\left(L\left(\Gamma_{i}\right), \frac{1}{S_{G_{i}}}\right) \quad i \in \text { All cycles }
$$

In this case, the shape optimisation of a closed section becomes a bi-objective mean cycle minimisation problem, in which the objectives are functions of the nodal coordinates.

\subsection{Special cases of shape optimisation}

In many instances, the required section strength is already determined and the aim is to find the shape of minimum mass that provides the required strength. In this case, the optimisation problem can be converted into a single objective problem where the required strength can be treated as a behavioral constraint on the state variable. For example, in the shape optimisation of a section for flexural rigidity, if the required second moment of area is predetermined, then the only objective is mass minimisation while the required second moment of area is treated as a constraint.

The present methodology can also be readily applied to the shape optimisation of steel sheeting profiles. Steel sheeting profiles can be treated as open sections with the two ends predetermined (for example as a result of being fixed on purlins). In this case, the problem becomes a single-pair shortest path problem as the source and destination nodes are fixed, which is much simpler than an all-pairs shortest path problem.

\subsection{Sizing optimisation}

In contrast to a shape optimisation problem, a sizing optimisation problem with discrete design variables is more difficult to solve than a similar problem with continuous design variables. However, structural optimisation methods employing the zero-one based decision making scheme are capable of dealing with the sizing optimisation problem using discrete variables $[34,35]$. In a sizing optimisation problem, there are a finite number of variables along with some constraints defining the boundary of the cross-section. Fig. 4 illustrates the variables of some standard sections. Depending on the problem at hand, each variable defined in Fig. 4 may or may not be used as a design variable. 

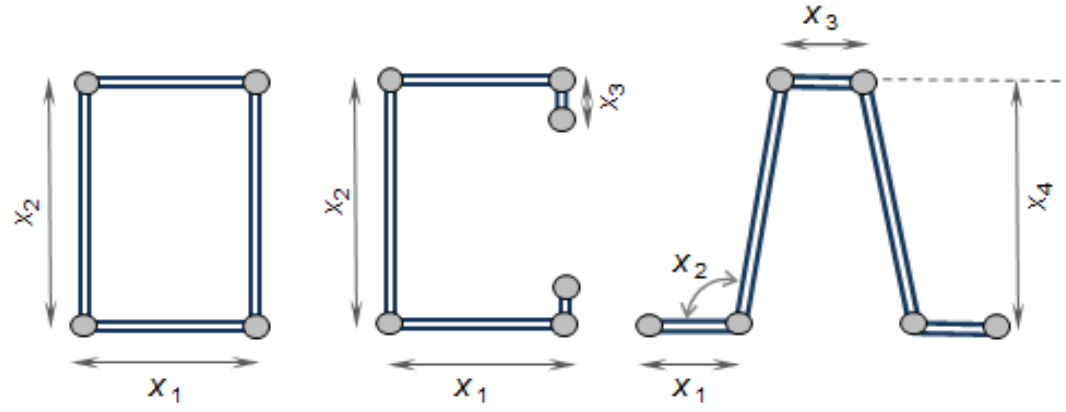

Fig. 4 Variables for standard sections of constant thickness

In order to define a graph model for dimension optimisation, the first step is to discretise the search space for each variable. To that end, each variable $x_{i}$ is bounded between $\left[\operatorname{Min}\left(x_{i}\right)\right.$, $\left.\operatorname{Max}\left(x_{i}\right)\right]$ with interval (accuracy) of $\varepsilon$, then each variable $x_{i}$ rests in the set of $\left[\operatorname{Min}\left(x_{i}\right)\right.$, $\left.\operatorname{Min}\left(x_{i}\right)+\varepsilon, \ldots, \operatorname{Max}\left(x_{i}\right)-\varepsilon, \operatorname{Max}\left(x_{i}\right)\right]$, which contains $m_{i}=\left(\left(\operatorname{Max}\left(x_{i}\right)-\operatorname{Min}\left(x_{i}\right)\right) / 2\right)+1$ possible conditions. If the number of variables is $v$, the graph corresponding to the sizing optimisation problem consists of $n=v+1$ nodes and $m=v \sum m_{i}$ edges as shown in Fig. 5 [28]. Any path through the graph represents a section with known dimensions. The objective functions are formulated in the same manner as those shown in the preceding sections on shape optimisation.

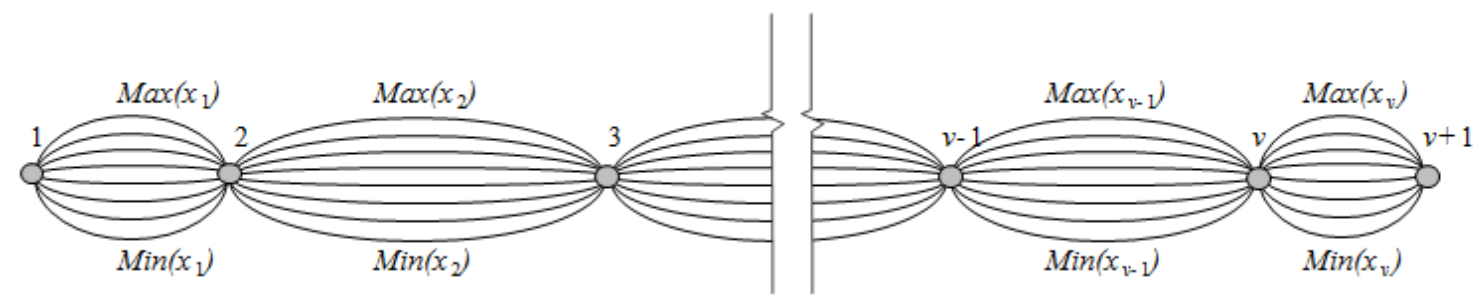

Fig. 5 A Graph for sizing optimisation

The problem of sizing optimisation becomes a multi-objective single-pair shortest path problem whose aim is to find the shortest path between a specific pair of nodes. Therefore, the integer programming formulation for sizing optimisation is the same as that for the shape optimisation of open sections. 


\section{$4 \quad$ Problem Formulations}

A multi-objective optimisation problem $f=\left(f_{1}, f_{2}, \ldots, f_{Q}\right)$ is a problem of finding a vector of decision variables that satisfies the constraints and optimises the vector function $f$ whose elements $f_{1}$ through $f_{Q}$ represent the $Q$ number of objective functions, which are usually in conflict with each other. In the present graph theory approach, the objective of the shortest path problem is to find a shape for the thin-walled steel section that has the minimum mass and the maximum strength. The optimisation problem can generally be formulated as follows:

$$
\begin{aligned}
& \min f=\left(W, \frac{1}{S_{G}}\right) \\
& \text { s.t. }\left\{\begin{array}{r}
\text { geometric constraints } \\
\text { strength constraints }
\end{array}\right.
\end{aligned}
$$

where $W$ is the section's mass. Depending on the problem at hand, a section strength $S_{\mathrm{G}}$ may mean the strength with respect to axial compression, flexural, torsional or shear action effects. In practice, the parameters that represent the section strength are mostly the second moments of area, the torsion constant or the cross-sectional area. Equation (14) is the basis of the shape optimisation problem.

Geometric constraints in shape optimisations of thin-walled steel sections may be governed by design standards, manufacturing and/or construction requirements. For example, the Australian cold-formed steel standard AS/NZ 4600 [33] may impose dimensional limitations, such as the maximum width to thickness ratio of the plate element.

In the present study, the optimum solution is the one that provides the best compromise between two potentially conflicting objectives of mass minimisation and strength maximisation. One approach to determining the solution is to find the Pareto-optimal set, or at least a good approximation of it.

Pareto optimality is an economics concept invented by Vilfredo Pareto (1848-1923) that finds applications in engineering. In a Pareto improvement, at least one objective is achieved without sacrificing any other objective. A solution is Pareto optimal when no further Pareto improvements can be made. A Pareto-optimal set is a set of Pareto optimal solutions. 
Having established a Pareto-optimal set, the final solution may be selected according to the personal intuition of the decision maker. The alternative approach is to formally assign weights or priorities to each objective before solving the problem so that the multi-objective optimisation problem is transformed into a single-objective problem (as the various objectives are combined into one through their weighted sum).

\section{$5 \quad$ Multi-objective Ant Colony Optimisation (ACO)}

A general ACO algorithm consists of three stages as shown in Fig. 6. In the first stage, the evaluation functions, pheromone trails and data are initialised. In the second stage, ants start constructing the solution using the random proportional rule. The third stage is to evaluate the solutions and update the pheromone trails according to their fitness values to help succeeding ants select a better path. The second and third stages cycle until all ants have finished constructing their solutions and the termination criterion is met.

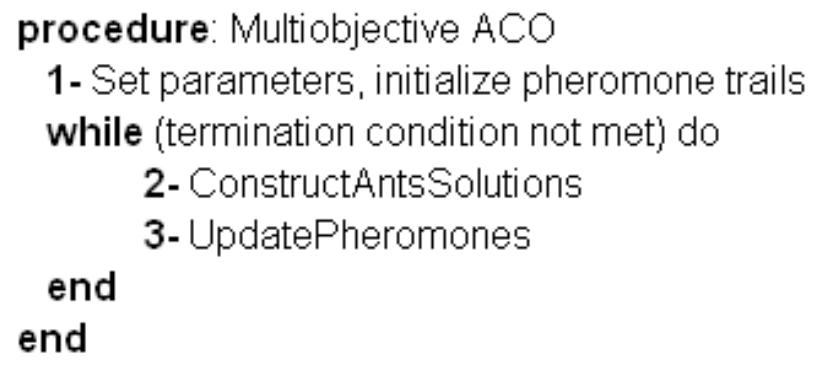

Fig. 6 Algorithm skeleton for multi-objective ACO

Multi-colony ACO algorithm [36] is used for multi-objective optimisation problems. There is an independent family of ants for each objective function, and the ants within each family search for optimum solutions to their assigned objective function. This search is carried out using a cooperation mechanism, where any information updated by an ant becomes available for all the other ants within the same family. Solutions proposed by a family are also transmitted to ants belonging to the other families, and the recipient ants then modify them to suit their respective objective functions. When all families have participated in constructing solutions, non-dominated solutions receive pheromone for the next iteration [37]. It enables finding several members of the Pareto optimal set in a single run instead of a series of runs, which is the case for some of the conventional stochastic processes. Computational results 
suggest that the multi-colony approach leads to improved performance when compared to the use of a single colony with single heuristic information.

For a multi-objective problem with $Q$ objectives, multiple pheromone information is defined for each objective, and weights are used to aggregate them into a single value for each family. Each edge $j$ in a solution $S_{q}$ for the objective function $f_{q}$ has its own pheromone matrix ${ }^{q} \tau_{S j}$ and heuristic information matrix ${ }^{q} \eta_{S j}$ that describe the desirability of choosing edge $j$. Given the construction graph, pheromone and heuristic information matrices, the probability with which ant $k$ chooses edge $j$ to its partial solution is:

$$
{ }^{k} p_{s j}=\frac{\left[\prod_{q=1}^{Q}\left({ }^{q} \tau_{S_{q j}}\right)^{\lambda_{q}}\right]^{\alpha} \cdot\left[\prod_{q=1}^{Q}\left({ }^{q} \eta_{S_{q j}}\right)^{\lambda_{q}}\right]^{\beta}}{\sum_{l \in{ }^{k} N_{s}}\left(\left[\prod_{q=1}^{Q}\left({ }^{q} \tau_{S_{q j}}\right)^{\lambda_{q}}\right]^{\alpha} \cdot\left[\prod_{q=1}^{Q}\left({ }^{q} \eta_{S_{q j}}\right)^{\lambda_{q}}\right]^{\beta}\right)}
$$

in which, ${ }^{k} N_{S}$ is the feasible neighbourhood of ant $k$, given the current state vector $S=\left\{S_{1}\right.$, $\left.S_{2}, \ldots, S_{Q}\right\} ; \alpha$ and $\beta$ are two parameters determining the relative influence of the pheromone trail and the heuristic information, and $\lambda_{q}$ is the value that weighs the relative importance of the $q^{\text {th }}$ objective function. Thus, the $q^{\text {th }}$ objective is not considered when $\lambda_{q}=0$, and it is the only one considered when $\lambda_{q}=1$.

In multiple ACO algorithm, the set of weight vectors that each colony applies in order to aggregate its multiple pheromone information represents a region in the objective space on which the colony focuses the search. For the bi-objective case, as represented by Eq. (14), a single value $\lambda$ is enough to define each weight vector $\{1-\lambda, \lambda\}$, as $Q=2$. In this case, Eq. (16) can be used in lieu of Eq. (15)

$$
{ }^{k} p_{s j}=\frac{\left[\left({ }^{1} \tau_{i j}\right)^{(1-\lambda)}\left({ }^{2} \tau_{i j}\right)^{\lambda}\right]^{\alpha} \cdot\left[\left({ }^{1} \eta_{i j}\right)^{(1-\lambda)}\left({ }^{2} \eta_{i j}\right)^{\lambda}\right]^{\beta}}{\sum_{l \in{ }^{k} N_{s}}\left(\left[\left({ }^{1} \tau_{i j}\right)^{(1-\lambda)}\left({ }^{2} \tau_{i j}\right)^{\lambda}\right]^{\alpha} \cdot\left[\left({ }^{1} \eta_{i j}\right)^{(1-\lambda)}\left({ }^{2} \eta_{i j}\right)^{\lambda}\right]^{\beta}\right)}
$$

In multi-objective problems, as in single objective ones, the iteration-best or best-so-far strategy can be used for pheromone update by taking the best solutions from a candidate set including all solutions found in the current iteration or since the start of the algorithm. In this case, the straightforward criterion is the Pareto optimality and thus the best solutions of the 
candidate set are those that are non-dominated, or in other words, those solutions that belong to the Pareto set. As each objective defines the solution components in a different way, the selected solutions update each pheromone matrix differently because each solution component has its own meaning for every pheromone matrix [36]. In this strategy, only the best solutions with respect to each objective are selected to update the pheromone information. Then, each pheromone matrix, associated with each objective, is updated by the solution with the best value for the corresponding objective. Therefore, the minimum number of ants allowed to update the pheromone information in a colony is equal to the number of objectives. Since an ant cannot update the pheromone information of more than one colony, each pheromone matrix focuses on one objective, thus the aggregation of all of them by means of a weight vector regulates the relative importance of each objective.

In the pheromone updating stage of multi-objective ACO, some special care must be taken to guarantee an acceptable convergence. For pheromone depositing, the only restriction is that the total solution cost cannot be used for this purpose, because the values of different objective functions are not comparable, and the amount of deposited pheromone must be ensured to be independent for each family. Otherwise, some objectives are implicitly considered to be more important than the others. With regard to the pheromone evaporation procedure, any method can be used although more efficient techniques would of course be preferred.

\subsection{Multi-objective ACO algorithm for shape optimisation}

The bi-objective ACO algorithm formulated for the present problem is a Max-Min Ant System (MMAS) algorithm [38], performed in the following steps:

\section{Stage 1- Initialise Data:}

In this stage, before starting the iterative part of the algorithm, the required data is initialised in the following manner.

1. Read instance: The mechanical properties of the steel, maximum cross-sectional dimensions allowed, and accuracy needed are defined.

2. Build construction graph: The grid pattern construction graph is formed in such a way that every node/edge on the graph is a potential node/edge of a cross-section. For this 
purpose, some geometrical, manufacturing and construction constraints, which may determine the shape boundaries, are applied to form the construction graph.

3. Represent ants: The number of families is equal to the number of objectives, i.e. two families for a bi-objective problem. The number of ants per family must be set in accordance with the termination criterion such that the exploration and exploitation processes are balanced [39]. In this algorithm, the number of ants for each family is set to be as many as the number of graph nodes.

4. Set heuristic information: If there is a preference for some forms or edge orientations, or if there are constraints on the shapes or edges, the arrays of the heuristic matrix corresponding to those constraints receive higher values that give them higher probability of being chosen. If there is no preference or constraint, heuristic matrices for each objective can be defined as $\left[{ }^{q} \eta_{i j}\right]=\left[d_{i j}\right]$, where $d_{i j}$ is the distance between nodes $i$ and $j$.

5. Set parameters: Parameters like $\alpha, \beta$ and $\lambda$, which determine the relative influence of the pheromone trail, the heuristic information, and the relative importance of each objective function, respectively, are determined. The evaporation rate $\rho \in(0,1]$ is defined for all colonies. The parameters $\alpha, \beta$ and $\rho$ are considered to be constant for all objectives. For MMAS, useful hints for defining efficient values for $\alpha, \beta$ and $\rho$ can be found in [39].

6. Initialise pheromone trails: MMAS limits the possible range of pheromone trail values to the interval $\left[{ }^{q} \tau_{\min },{ }^{q} \tau_{\max }\right]$. Also, the pheromone trails are initialised to the upper pheromone trail limit, which, together with a small pheromone evaporation rate, increases the exploration of tours at the start of the search [40]. As a good estimate, the lower level is set to be ${ }^{q} \tau_{\min }={ }^{q} \tau_{\max } / a$, in which the variable $a$ is a parameter that can be calculated based on the quality of solutions [38].

\section{$\underline{\text { Stage 2- Construct Ant Solutions }}$}

The stage of construction graph continues iteratively until the termination criterion is met. There is no general termination criterion applicable for all ACO algorithms. Depending on the optimisation problem, some criteria such as the maximum CPU time, the maximum number of solutions generated, the percentage deviation from a lower/upper bound from the optimum, and the maximum number of iterations without improvement in solution quality, or a combination of them can be set as the termination criterion. This stage of the algorithm is performed as follows: 
1. Empty ant's memory: All the edges and nodes of the graph are marked as unselected.

2. Place ants on initial positions: For the bi-objective problem of shape optimisation, two ants are allocated to each node: one ant belonging to the mass minimisation family, and the other to the strength maximisation family (minimisation of inverse strength).

3. Construct solutions: Ants start constructing their solutions independently using Eq. (16). For constructing the trails for each problem, the ants have to comply with the corresponding constraints. Ants select nodes one after another to create a path (or cycle) which represents a cross-section. In order to make the paths (or cycles) more realistic, constraints on the ants' movement and/or nodes selection can be applied. For example, in order to obtain smooth and realistic bends, and control the minimum size of the bends, there can be a constraint such that ants are only allowed to select the nodes whose adjacent nodes have not been selected yet.

4. Save solutions information: The costs for all solution are explored; best-so-far and iteration-best solution solutions for each family are determined.

\section{$\underline{\text { Stage 3- Update Pheromone Trails }}$}

Iterative pheromone update continues until the termination criterion is met. This stage is performed in two steps as follows:

1. Pheromone evaporation: The pheromone evaporation on all edges and for all objectives is implemented by:

$$
{ }^{q} \tau_{i j} \leftarrow(1-\rho)^{q} \tau_{i j}
$$

2. Pheromone deposit: The pheromone evaporation is followed by the deposit of new pheromone on all edges and for all objectives as follows:

$$
{ }^{q} \tau_{i j} \leftarrow{ }^{q} \tau_{i j}+\Delta^{q . b e s t} \tau_{i j}
$$

where ${ }^{q . b e s t} \tau_{i j}=1 /{ }^{q . b e s t} C$, and ${ }^{q . b e s t} C$ is the cost of best-so-far or iteration-best solution for the objective $q$. Both best-so-far and iteration-best ants are allowed to deposit pheromone, but only for their own pheromone matrix and independently from the other families. Experimental results indicate that for small instances it may be best to use only iterationbest pheromone updates, while for large ones with several hundreds of nodes the best 
performance is obtained by giving an increasingly stronger emphasis to the best-so-far tour $[38,39]$.

\subsection{Multi-objective ACO algorithm for sizing optimisation}

The procedure of the ACO algorithm for sizing optimisation is similar to that for shape optimisation described in the preceding section. However, there are some differences in certain steps as detailed in the following:

- Build construction graph: The construction graph is formed in such a way that the number of nodes is equal to the number of design variables plus one, and the number of edges is determined according to the maximum and minimum possible values for each variable and the accuracy required.

- Represent ants: If the number of graph's edges is $m$ and the number of nodes is $n$, the number of ants for each family is set to be the nearest integer to $m /(n-1)$.

- Set heuristic information: If there is a preference for some sizes or if there are constraints, the arrays of the heuristic matrix corresponding to those sizes or constraints receive higher values that give them higher probability of being chosen. If there is no preference or constraint, heuristic matrices for objectives can be removed from the computation process $(\beta=0)$.

- Place ants on initial positions: Two families of ants are placed on the source node of the graph. Since the algorithm deals with a single-pair shortest path problem, the ants always restart their search from the source node in each iteration.

\section{Numerical Examples}

\subsection{Verification example}

In order to verify the present methodology, a closed thin-walled section is optimised for minimum mass (first objective) and maximum second moments of area about both principal axes (second objective). The section has $1 \mathrm{~mm}$ wall thickness and is subject to a constraint that the elastic section modulus cannot exceed $660 \mathrm{~mm}^{3}$ by more than $5 \%$. In this case, the globally optimum section is known to be a circular hollow section with a diameter of $30 \mathrm{~mm}$ (accurate to the millimeter). 

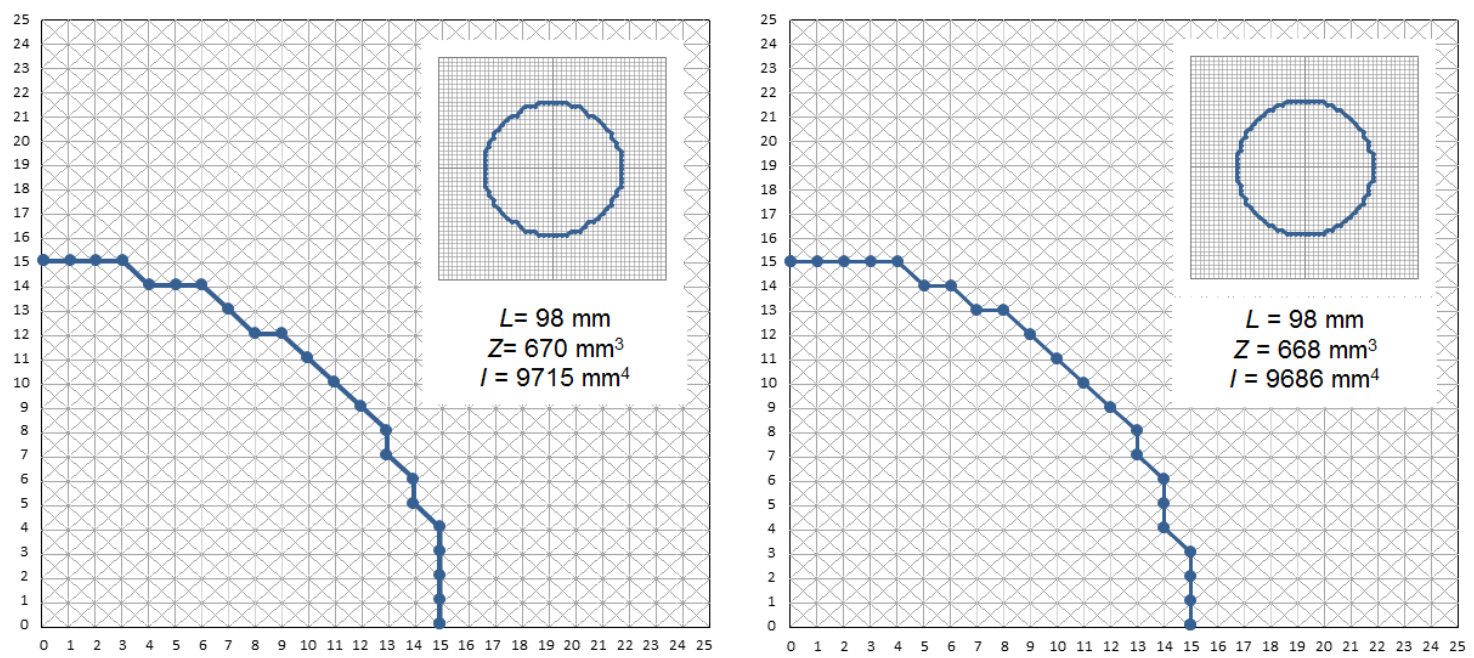

Fig. 7 Two best Pareto-optimal solutions for verification of the methodology

A $25 \mathrm{~mm}$ by $25 \mathrm{~mm}$ grid graph with diagonal edges and minimum resolution of $\varepsilon=1 \mathrm{~mm}$ is defined for constructing a quarter of the section. Fig. 7 shows the two best "optimum" shapes (the first two members of the Pareto-optimal set).

\subsection{Shape optimization of open and closed sections for elastic section modulus}

In this example, an open and a closed thin-walled section under transverse loading are optimised for minimum mass (first objective) and maximum elastic section modulus about the major axis (second objective). The geometric data and constraints are:

- Wall thickness: $t=1 \mathrm{~mm}$

- Maximum cross-sectional height: $100 \mathrm{~mm}$.

- Maximum cross-sectional width: $80 \mathrm{~mm}$.

- Maximum ratio of flat width to wall thickness, $b / t: 60$

- Minimum resolution (accuracy): $\varepsilon=2 \mathrm{~mm}$

- Closed sections are doubly-symmetric and open sections are singly symmetric

The material properties and the strength constraints are as follows:

- Elastic modulus: $200 \mathrm{GPa}$

- Yield stress: $300 \mathrm{MPa}$

- Poisson's ratio: 0.3

- Minimum second moment of area about the major axis: $6.6 \times 10^{5} \mathrm{~mm}^{4}$

- Maximum second moment of area about the minor axis: $1.32 \times 10^{5} \mathrm{~mm}^{4}$

The present bi-objective optimization problems are summarised in Table 1. 
Table 1. Optimisation problem of Example 6.2

\begin{tabular}{|c|c|c|}
\hline & Open Cross-sections & Closed Cross-sections \\
\hline Objective Function & $\begin{array}{c}\min f_{P}=\left(L\left(P_{i}\right), \frac{1}{S_{f_{i}}}\right) \\
\Rightarrow \min f=\left(\sum_{i, j \in V}{ }^{1} c_{i j} e_{i j}, \sum_{i, j \in V}{ }^{2} c_{i j} e_{i j}\right)\end{array}$ & $\begin{array}{c}\min f_{\Gamma}=\left(L\left(\Gamma_{i}\right), \frac{1}{S_{f_{i}}}\right) \\
\Rightarrow \min f=\left(\sum_{i, j \in V}{ }^{1} c_{i j} e_{i j}, \sum_{i, j \in V}{ }^{2} c_{i j} e_{i j}\right)\end{array}$ \\
\hline \multirow[b]{2}{*}{$\begin{array}{l}\text { Behavioral } \\
\text { Constraints }\end{array}$} & \multicolumn{2}{|c|}{$\forall e_{i j} \in E: e_{i j}= \begin{cases}1 & \text { if Edge } e_{i j} \text { is chosen } \\
0 & \text { if Edge } e_{i j} \text { is notchosen }\end{cases}$} \\
\hline & $\begin{array}{c}\forall i \in N-\{s, t\}: \sum_{j} e_{i j}-\sum_{k} e_{k i}=0 \\
\forall i, j \in N: \sum_{i} e_{s i}-\sum_{j} e_{j s}=1 \\
\forall i, j \in N: \sum_{i} e_{i t}-\sum_{j} e_{t j}=1\end{array}$ & $\forall i \in N: \sum_{j} e_{i j}-\sum_{k} e_{k i}=0$ \\
\hline \multirow{2}{*}{$\begin{array}{l}\text { Strength and } \\
\text { Serviceability } \\
\text { Constraints }\end{array}$} & \multicolumn{2}{|c|}{$I_{x x} \geq 6.6 \times 10^{5} \mathrm{~mm}^{4}$} \\
\hline & \multicolumn{2}{|c|}{$I_{z z} \leq 1.32 \times 10^{5} \mathrm{~mm}^{4}$} \\
\hline Geometric Constraint & \multicolumn{2}{|c|}{$\frac{b}{t} \leq 60$} \\
\hline
\end{tabular}

Since the closed sections are doubly-symmetric and the open sections are mono-symmetric, only a quarter of the closed section and one half of the open section are modeled. The construction graph for the closed section problem is $40 \mathrm{~mm}$ by $50 \mathrm{~mm}$, and for the open section problem is $80 \mathrm{~mm}$ by $50 \mathrm{~mm}$.

In the case of the doubly symmetric graph (closed section), ants, initially located at the nodes along the vertical axis of symmetry (the source nodes), move towards the nodes along the horizontal axis of symmetry (the destination nodes). There is no need for ants to be initialised at all nodes since the source nodes and the destination nodes of optimum solutions must lie on the coordinate axes, resulting in a reduced number of ants required to search the solutions. In the case of the singly symmetric graph (open section), ants only need to be initialised at the nodes along the axis of symmetry as the source nodes. Ants are not allowed to choose another node on the axis of symmetry in order to avoid an invalid open section.

The parameters applied to the ACO algorithm are shown in Table 2. In each step of the algorithm, basic analysis is required to calculate the geometrical properties of the obtained 
sections based on the nodal coordinates. In order to obtain smoother bends, ants are only allowed to select a node whose adjacent nodes have not been selected yet.

Table 2: Parameters for ACO Algorithm for Example 6.2

\begin{tabular}{ccccccccc}
\hline $\begin{array}{c}\text { Number } \\
\text { of ants }\end{array}$ & $\alpha$ & $\beta$ & $\lambda$ & $\rho$ & ${ }^{1} \tau_{\min }$ & ${ }^{1} \tau_{\max }$ & ${ }^{2} \tau_{\min }$ & ${ }^{2} \tau_{\max }$ \\
\hline 1200 & 3 & 5 & 0.5 & 0.01 & 4.4 & 9.6 & 1.1 & 4.5 \\
\hline
\end{tabular}

The termination criterion for this problem is met when the improvement in the solution quality is less than $2 \%$ after ten consecutive iterations. The optimum shapes are the best-sofar solutions. Figs. 8 and 9 show the three best "optimum" shapes (i.e. the first three members of the Pareto-optimal set), obtained after 893 iterations, for a quarter of the closed section and one half of the open section, respectively.

In order to accentuate the difference between the obtained open sections, Fig. 9 has been drawn by moving all the sections towards the $y$ axis so that the starting and ending points lie on the $x$ and $y$ axes, respectively. The drawn paths are therefore not exactly the paths chosen by ants, but are the equivalent paths having the same characteristics. 

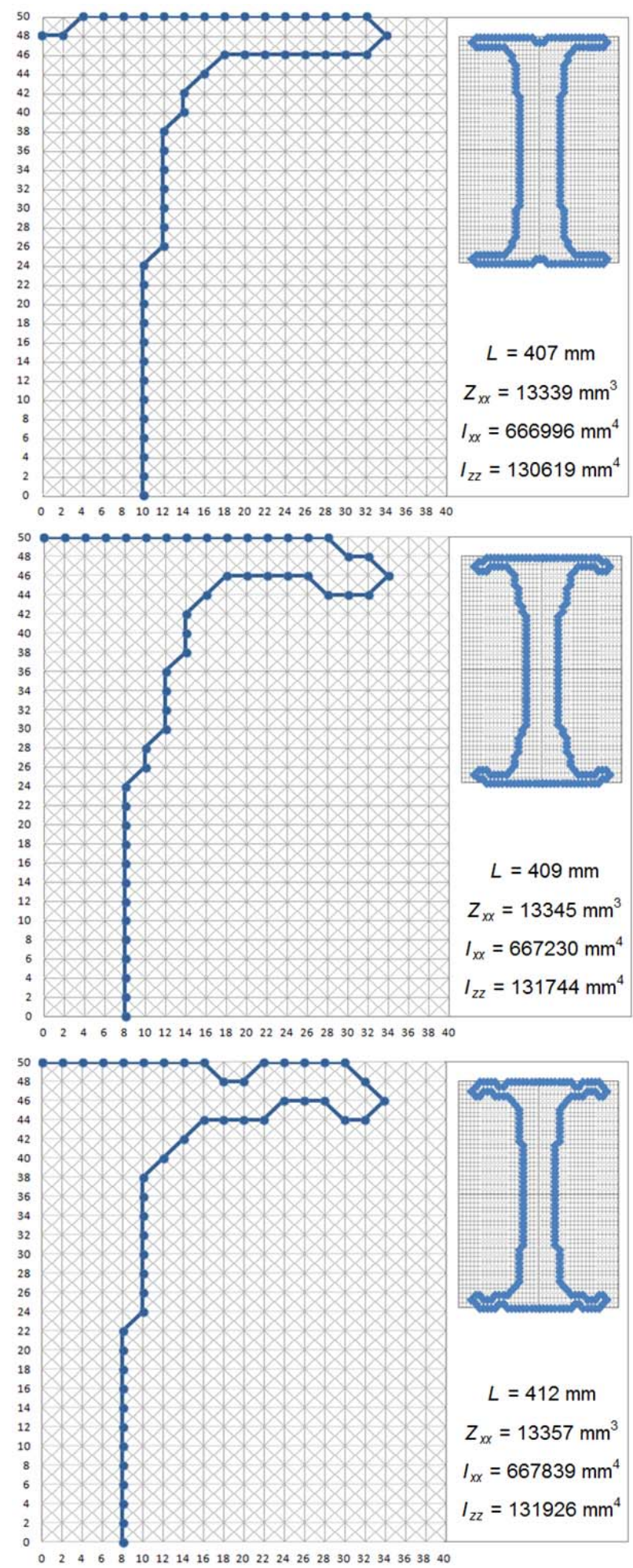

Fig. 8 The three first Pareto-optimal solutions for the closed section 

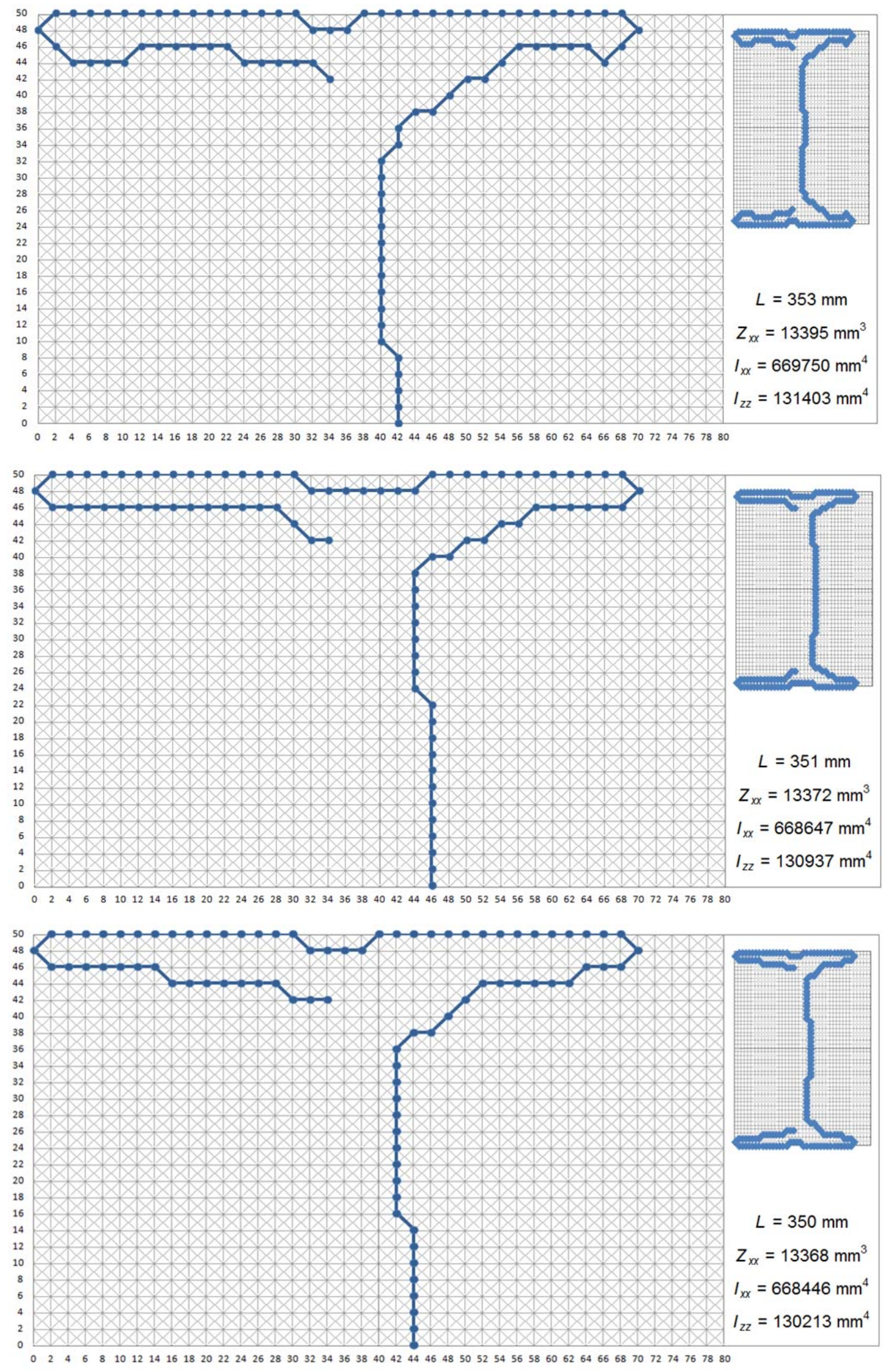

Fig. 9 The three first Pareto-optimal solutions for the open section 


\subsection{Sizing optimization of trapezoidal roof sheeting}

Consider a trapezoidal steel roof-sheeting panel that is used to support solar modules as shown in Fig. 10. The aim is to optimise the dimensions of the trapezoidal panel in order to minimise its mass (first objective) and maximise its elastic section modulus about the major axis (second objective). Four design variables are involved in the optimization: rib height $\left(V_{1}\right)$, rib width $\left(H_{1}\right)$, rib slope $(\theta)$ and flat pan midpoint height $\left(V_{2}\right)$.

The known geometric data and constraints are given in the following:

- The sheeting thickness is $0.6 \mathrm{~mm}$.

- The panel is fixed at nodes A and B, and there are no vertical displacements at points C, D, E, and F.

- The span $W$ between supports A and B is $1000 \mathrm{~mm}$.

- The width of the flat pan $\mathrm{H}_{2}$ is $400 \mathrm{~mm}$.

- The rib height $V_{1}$ may range from 15 to $50 \mathrm{~mm}$.

- The maximum flat pan midpoint height $V_{2}$ is $20 \mathrm{~mm}$.

- Maximum flat width to wall thickness ratio is 500 .

- Minimum resolution: $1 \mathrm{~mm}$ for $V_{1}, H_{1}$ and $V_{2}$; and $1^{\circ}$ for $\theta$

The material properties and the strength constraint are as follows:

- Elastic modulus: $200 \mathrm{GPa}$

- Yield stress: $300 \mathrm{MPa}$

- Poisson's ratio: 0.3

- Minimum second moment of area: $2 \times 10^{4} \mathrm{~mm}^{4}$

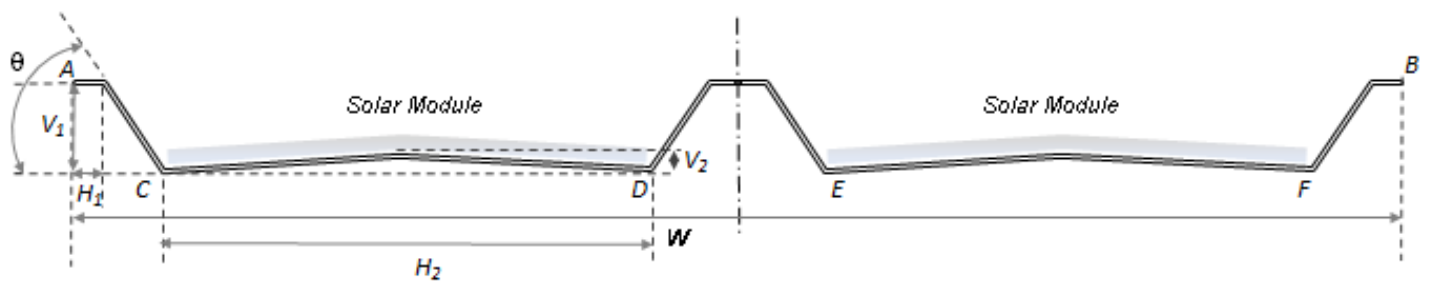

Fig. 10 Geometric dimensions of roof sheeting 
The single-pair shortest path problem is summarised in Table 3. The construction graph for this problem consists of five nodes as shown in Fig. 11. The parameters applied to the ACO algorithm are shown in Table 4. In each step, structural analysis is required to determine the geometric properties and action effects for the obtained sections.

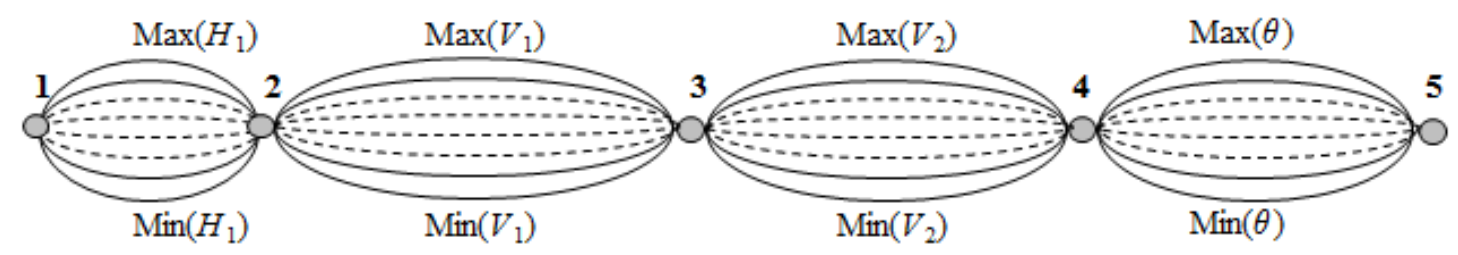

Fig. 11 Construction Graph for Sizing Optimization

Table 3: Optimization problem of Example 6.3

\begin{tabular}{|c|c|}
\hline \multirow{2}{*}{ Objective Function } & $f\left(V_{1}, H_{1}, V_{2}, \theta\right)$ \\
& $\min f_{P}=\left(L\left(P_{i}\right), \frac{1}{S_{f_{i}}}\right)$ \\
\hline & $\Rightarrow \min f=\left(\sum_{i, j \in V}{ }^{1} c_{i j} e_{i j}, \sum_{i, j \in V}{ }^{2} c_{i j} e_{i j}\right)$ \\
Behavioral & $\forall i \in N-\{s, t\}: \sum_{j} e_{i j}-\sum_{k} e_{k i}=0$ \\
Constraints & $\forall i, j \in N: \sum_{i} e_{s i}-\sum_{j} e_{j s}=1$ \\
& $\forall i, j \in N: \sum_{i} e_{i t}-\sum_{j} e_{t j}=1$ \\
\hline Serviceability & $I_{x x} \geq 2.0 \times 10^{4} \mathrm{~mm}^{4}$ \\
\hline Constraint & $\frac{b}{t} \leq 500$ \\
& $15 \mathrm{~mm} \leq V_{1} \leq 50 \mathrm{~mm}$ \\
Geometric & $0 \leq V_{2} \leq 20 \mathrm{~mm}$ \\
Constraints & $H_{2}=400 \mathrm{~mm}$ \\
\hline
\end{tabular}

Table 4: Parameters for ACO Algorithm for Example 6.3

\begin{tabular}{ccccccccc}
\hline $\begin{array}{c}\text { Number } \\
\text { of ants }\end{array}$ & $\mathrm{A}$ & $\beta$ & $\lambda$ & $\rho$ & ${ }^{1} \tau_{\min }$ & ${ }^{1} \tau_{\max }$ & ${ }^{2} \tau_{\min }$ & ${ }^{2} \tau_{\max }$ \\
\hline 5 & 3 & 5 & 0.5 & 0.05 & 3.1 & 8.9 & 0.9 & 4.1 \\
\hline
\end{tabular}


The termination criterion is met when the improvement in the solution quality is less than $2 \%$ ufter ten consecutive iterations. The first three solutions in the Pareto optimal set, obtained ifter 419 iterations, are shown in Table 5.

Table 5: Three Pareto-optimal solutions for Example 6.3

\begin{tabular}{ccccccc}
\hline $\begin{array}{c}H_{1} \\
(\mathrm{~mm})\end{array}$ & $\begin{array}{c}V_{1} \\
(\mathrm{~mm})\end{array}$ & $\begin{array}{c}V_{2} \\
(\mathrm{~mm})\end{array}$ & $\begin{array}{c}\theta \\
(\mathrm{deg})\end{array}$ & $\begin{array}{c}I \\
\left(\mathrm{~mm}^{4}\right)\end{array}$ & $\begin{array}{c}Z \\
\left(\mathrm{~mm}^{3}\right)\end{array}$ & $\begin{array}{c}\text { Mass } \\
(\mathrm{g})\end{array}$ \\
\hline 17 & 19 & 17 & 70 & 20026 & 2189 & 4866 \\
18 & 20 & 17 & 72 & 20748 & 2253 & 4991 \\
18 & 20 & 17 & 72 & 20473 & 2236 & 4961 \\
\hline
\end{tabular}

\section{$7 \quad$ Summary and concluding remarks}

A graph theory based method is a powerful means to represent a structural system so that its geometry and topology can be understood clearly. The developments of some robust metaheuristics such as ant colony algorithms in recent decades have enabled the analyst to deal with large graph theory based problems. This paper presents a new methodology for shape and sizing optimisations of thin-walled steel sections using the graph theory approach.

The optimisation problem is defined as a multi-objective problem that aims to minimise the mass and maximise the section strength. The shape optimisation of an open section is treated as a multi-objective all-pairs shortest path problem, while that of a closed section is treated as a multi-objective minimum mean cycle problem. The sizing optimisation of a predetermined shape is treated as a multi-objective single-pair shortest path problem.

Ant colony based optimisation algorithms are among of the most robust meta-heuristics for solving large combinatorial optimization problems which can be reduced to finding the shortest paths through graph theory models. The two conflicting objectives of mass minimisation and strength maximisation are handled using the Pareto-optimal set. This paper has demonstrated the applications of the resulting algorithms to shape and sizing 
optimizations of open and closed thin-walled steel sections including roof sheeting, accounting for geometric and strength constraints.

\section{Acknowledgment}

Funding of this research project was provided by the Bluescope Steel Metallurgy Centre at the University of Wollongong, sponsored by the Faculty of Engineering and Bluescope Steel Limited. Any opinions expressed are those of the authors alone.

\section{References}

1. Ding S, Daniel WJT, Yuan J, Zhang Y. Making roll forming flexible - Introduction to chain forming. Joint Symp Innovative Tube and Pipe Manufacturing and Forming, Yokohama, Japan, 2001: 341-346.

2. Schafer BW. Review: The Direct Strength Method of cold-formed steel member design. J Construct Steel Res 2008; 64(7-8): 766-778.

3. Karim A, Adeli H. Global optimum design of cold-formed steel hat-shape beams. ThinWalled Struct 1999; 35(4): 275-288.

4. Lee J, Kim SM, Park HS, Woo BH. Optimum design of cold-formed steel channel beams using micro Genetic Algorithm. Eng Struct 2005; 27(1): 17-24.

5. Tran T, Li LY. Global optimisation of cold-formed steel channel sections. Thin-Walled Struct 2006; 44(4): 399-406.

6. Lu W, Mäkeläinen P. Fuzzy optimisation of cold-formed steel sheeting using genetic algorithms. J Construct Steel Res 2006; 62(12): 1276-1281.

7. Magnucki K, Rodak M, Lewiński J. Optimisation of mono- and anti-symmetrical Isections of cold-formed thin-walled beams. Thin-Walled Struct 2006; 44(8): 832-836.

8. Kasperska RJ, Magnucki K, Ostwald M. Bicriteria optimisation of cold-formed thinwalled beams with monosymmetrical open cross sections under pure bending. ThinWalled Structures 2007; 45(6): 563-572.

9. Magnucki K, Paczos P. Theoretical shape optimisation of cold-formed thin-walled channel beams with drop flanges in pure bending. J Construct Steel Res 2009; 65(8-9): 1731-1737. 
10. Pastor MM, Casafont M, Chillarón E, Lusa A, Roure F, Somalo MR. Optimisation of cold-formed steel pallet racking cross-sections for flexural-torsional buckling with constraints on the geometry. Eng Struct 2009; 31(11): 2711-2722.

11. Magnucka-Blandzi E. Effective shaping of cold-formed thin-walled channel beams with double-box flanges in pure bending. Thin-Walled Struct 2011; 49(1): 121-128.

12. Magnucka-Blandzi E, Magnucki K. Buckling and optimal design of cold-formed thinwalled beams: Review of selected problems. Thin-Walled Struct 2011; 49(5): 554-561.

13. Lee CL, Mioduchowski A, Faulkner MG. Optimisation of corrugated claddings. J Struct Eng 1995; 121(8): 1190-1196.

14. Vinot P, Cogan S, Piranda J. Shape optimisation of thin-walled beam-like structures. Thin-Walled Struct 2001; 39(7): 611-630.

15. Leng J, Guest JK, Schafer BW. Shape optimisation of cold-formed steel columns. ThinWalled Struct 2011; 49(12): 1492-1503.

16. Gilbert BP, Teh LH, Guan. H. Self-shape optimisation principles: Optimisation of section capacity for thin-walled profiles. Thin-Walled Struct 2012; 60: 194-204.

17. Gilbert BP, M. Savoyat TJ, Teh LH. Self-shape optimisation application: Optimisation of cold-formed steel columns. Thin-Walled Struct 2012; 173: 184-204.

18. Spillers WR. Application of topology in structural analysis, J Struct Div ASCE 1963; 89: 301-313.

19. Kaveh A. Structural mechanics: Graph and matrix methods 3rd ed. 2004: Research Studies Press (John Wiley).

20. Kaveh A. Optimal analysis of structures by concepts of symmetry and regularity. 2013: Springer Verlag.

21. Descamps B, Coelho RF. Graph theory in evolutionary truss design optimisation. In: Gandomi AS, Yang XS, Talatahari S, Alavi AH, editors. Metaheuristic Applications in Structures and Infrastructures. Amsterdam: Elsevier; 2013: 241-268.

22. Kaveh A, Kalatjari V. Topology optimisation of trusses using genetic algorithm, force method and graph theory. Int J Numer Meth Eng 2003; 58: 771- 791.

23. Wang SY, Tai K. Graph representation for structural topology optimisation using genetic algorithms. Comput Struct 2004; 82: 1609-1622. 
24. Kaveh A, Sharafi P. Ant colony optimisation for finding medians of weighted graphs. Eng Comp 2008; 25(2): 102-120.

25. Kaveh A, Sharafi P. Optimal priority functions for profile reduction using ant colony optimisation. Finite Elements in Analysis and Design 2008; 44(3): 131-138.

26. Kaveh A, Hassani B, Shojaee S, Tavakkoli SM. Structural topology optimization using ant colony methodology. Eng Struct 2008; 30(9): 2559-2565.

27. Dorigo M, Birattari M, Stutzle T. Ant colony optimization - Artificial ants as a computational intelligence technique. IEEE Computational Intelligence Magazine 2006; 1(4): 28-39.

28. Sharafi P, Hadi MNS, Teh LH. Heuristic approach for optimum cost and layout design of 3D reinforced concrete frames. J Struct Eng 2012; 138(7): 853-863.

29. Sharafi P, Hadi MNS, Teh LH. Geometric design optimisation for dynamic response problem of continuous reinforced concrete beams. J Comput Civ Eng 2014; 28(2): 202209.

30. Lawler EL. Combinatorial Optimisation: Networks and Matroids. 2001: Dover Publications.

31. Reinhardt LB, Pisinger D. Multi-objective and multi-constrained non-additive shortest path problems. Computers \& Operations Research 2011; 38(3): 605-616.

32. Karp RM. A characterization of the minimum cycle mean in a digraph. Discrete Math 1978; 23(3): 309-311.

33. AS/NZ4600. Cold-formed steel structures 2005, Standards Australia Limited/ Standards New Zealand: Sydney.

34. Steven GP, Li Q, Xie YM. Multicriteria optimisation that minimises maximum stress and maximises stiffness. Comput Struct 2002; 80(27-30): 2433-2448.

35. Burns SA. Recent advances in optimal structural design. 2002: American Society of Civil Engineers, Structural Engineering Institute. Technical Committee on Optimal Structural Design, Reston, Virginia.

36. Iredi S, Merkle D, Middendorf M. Bi-criterion optimization with multi colony ant algorithms. Evolutionary Multi-Criterion Optimization, Zitzler et al., eds. 2001, Springer Berlin / Heidelberg, 359-372. 
37. Coello CAC, Lamont GB, Van Veldhuizen DA. Evolutionary algorithms for solving multi-objective problems. 2007: Springer.

38. Stutzle T, Hoos H. Max-Min Ant System and local search for the traveling salesman problem. IEEE Int Conf Evolutionary Computation, 1997.

39. Dorigo M. Ant colony optimization and swarm intelligence: 5th International Workshop, ANTS 2006, Brussels, Belgium, September 4-7, 2006.

40. Dorigo M, Stützle T. Ant colony optimization. 2004: MIT Press. 